\title{
NONLINEAR DYNAMICAL BOUNDARY-VALUE PROBLEM OF HYDROGEN THERMAL DESORPTION
}

\author{
YU. V. ZAIKA and I. A. CHERNOV
}

Received 25 March 2002

\begin{abstract}
The nonlinear boundary-value problem for the diffusion equation, which models gas interaction with solids, is considered. The model includes diffusion and the sorption/desorption processes on the surface, which leads to dynamical nonlinear boundary conditions. The boundary-value problem is reduced to an integrodifferential equation of a special kind; existence and uniqueness of the classical (differentiable) solution theorems are proved. The results of numerical experiments are presented.
\end{abstract}

2000 Mathematics Subject Classification: 35K60, $65 \mathrm{Z} 05$.

1. Introduction. The hydrogen presence in widely used construction materials often leads to the worsening of their operational state. Due to the importance of ecological safety, this must be taken into consideration when developing chemical industry and power engineering objects. Interest in hydrogen power engineering has grown in the recent time as it is ecologically safe. Thus transport and storage problems appear. All that has defined a growing interest to hydrogen interaction with different solid materials [1, 3, 4, 5]. Serious experimental and theoretical elaborations in this area are hardly possible without mathematical modeling. Numerical experiments allow to choose most adequate models with respect to experimental data, help to improve the understanding of different mechanisms and stages of the process, reduce needs for costly experiments, and estimate some parameters.

In this paper, a widely used experimental method of thermodesorption spectrometry will be considered (TDS) $[4,5]$. Here is its brief description: a plate from studied material is placed under hydrogen pressure. A plate is electrically heated to increase the rates of adsorbtion/desorbtion and diffusion. When balance concentration is obtained, the plate is cooled (turning electric heating off). The rates of mentioned processes abruptly decrease. Keeping vacuum around a plate, it is slowly heated again. The hydrogen desorption flux from the surface is estimated using mass spectrometer.

2. Mathematical model. Let $c(t, x)$ be the concentration of dissolved (atomic) hydrogen inside the plate $(t \geq 0, x \in[0, \ell])$. Initial data is determined by the fact that the plate had been saturated with the gas $c(0, x)=\bar{c}_{0}=$ const. 
In the area $Q_{t_{*}}=\left(0, t_{*}\right) \times(0, \ell)$, concentration satisfies the diffusion equation

$$
c_{t}(t, x)=D(T) c_{x x}(t, x)
$$

Here, $t_{*}$ is the duration of the TDS experiment, $\ell$ is the width of the plate, $D$ is the diffusion coefficient, and $T=T(t)$ represents the temperature. Limit values of $T$ are known: $T(t) \in\left[T^{-}, T^{+}\right], 0<T^{-}<T^{+}$, and $T(\cdot) \in C^{1}\left[0, t_{*}\right]$. Linear heating is often used (in the area $\left[T^{-}, T^{+}\right]$). For hydrogen at usual range of pressure, concentration, and temperature, the dependence of all parameters on $T$ is well described by Arrhenius rule: $D(T)=D_{0} \exp \left\{-E_{D} /[R T]\right\}$. Later in this paper $D(t)=D(T(t))$.

We describe the boundary conditions. Considering physical and chemical processes on the surface, the following dynamical conditions will be used [4]:

$$
\dot{q}_{0, \ell}(t)=\mu s(T) p(t)-b(T) q_{0, \ell}^{2}(t) \pm\left. D(T) c_{x}(t, x)\right|_{x=0, \ell} .
$$

This is a differential equation for surface concentrations $q_{0}(t), q_{\ell}(t)$, on both faces of the plate: $x=0, x=\ell$. Hydrogen atoms form molecules and desorb from the surface. The density of desorption flux for hydrogen depends quadratically on the concentration of atoms on the face

$$
J_{0, \ell}(t)=b(t) q_{0, \ell}^{2}(t), \quad b(t)=b(T(t)), b(t)=b_{0} \exp \left\{-\frac{E_{b}}{[R T(t)]}\right\} .
$$

Pressure $p(t)$ of gas hydrogen makes some amount of gas to return to the surface-it defines the first term in the right part of (2.2) $(\mu, s(T)$ are the physical constants). The last term in (2.2) defines the diffusion flux of hydrogen atoms from the deep to the surface. The experiment is symmetrical

$q(t)=q_{0}(t)=q_{\ell}(t), \quad J(t)=J_{0}(t)=J_{\ell}(t), \quad c(t, x)=c(t, \ell-x), \quad x \in[0, \ell]$.

The pressure is measured as

$$
p(t)=\theta_{1} \int_{0}^{t} J(\tau) \exp \left\{\frac{(\tau-t)}{\theta_{0}}\right\} d \tau
$$

constants $\theta_{i}$ are defined by technical details of the experimental equipment.

The density of desorption flux can be found from the pressure $p(t)$ for all $t \geq 0, J(t)=\left(\dot{p}(t)+p(t) / \theta_{0}\right) / \theta_{1}$.

If the vacuum system is powerful, the hydrogen return to the surface can be considered negligibly small. As all processes are symmetrical with respect to 
the middle of the plate, later in this paper we consider one equation instead of (2.2)

$$
\dot{q}(t)=-b(t) q^{2}(t)+D(t) c_{x}(t, 0) .
$$

The process of hydrogen remissing is considered fast enough, so linear connection between surface and subsurface concentrations can be efficiently used

$$
c(t, 0)=c_{0}(t)=g(t) q(t), \quad g(t)=g_{0} \exp \left\{-\frac{E_{g}}{[R T(t)]}\right\} .
$$

In this paper, the dependence of parameter on temperature is not important.

So the model of TDS experiment looks like

$$
\begin{aligned}
c_{t}(t, x) & =D(t) c_{x x}(t, x), \quad(t, x) \in Q_{t_{*}}, \\
c(0, x) & =\bar{c}_{0}, \quad c(t, x)=c(t, \ell-x), \quad x \in[0, \ell], \\
c(t, 0) & =g(t) q(t), \quad \dot{q}(t)=-b(t) q^{2}(t)+D(t) c_{x}(t, 0) .
\end{aligned}
$$

The main specificity of this boundary-value problem is in nonlinear dynamical boundary conditions.

More general problem from the viewpoint of generalized solutions has been studied in [8]. Some algorithms of parametric identification of hydrogen penetration models for stratified materials can be found in [3, 9, 10]. In this paper, the existence of classical solution of the given problem will be studied.

To simplify mathematical operations on the problem, we exclude the variable $q$ and consider new time $t^{\prime}=\int_{0}^{t} D(\tau) d \tau$. New time will be represented by the same letter $t$. After these transforms, the problem will be

$$
\begin{aligned}
c_{t}(t, x) & =c_{x x}(t, x), \quad(t, x) \in Q_{t_{*}}, \\
c(0, x) & =\bar{c}_{0}=\mathrm{const}, \quad x \in[0, \ell], \\
\dot{c}_{0}(t) & =-\alpha_{1}(t) c_{0}^{2}(t)+\alpha_{2}(t) c_{0}(t)+g(t) c_{x}(t, 0), \\
c_{0}(t) & =c(t, 0), \quad \alpha_{1}(t)=\frac{b}{D g}, \quad \alpha_{2}(t)=\frac{\dot{g}}{g}, \\
c(t, x) & =c(t, \ell-x), \quad x \in[0, \ell] .
\end{aligned}
$$

3. Reducing the problem to an integrodifferential equation. Let $C^{1,2}\left(\bar{Q}_{t_{*}}\right)$ be a space of functions on $\bar{Q}_{t_{*}}=\left[0, t_{*}\right] \times[0, \ell]$, which has continuous partial derivatives $\partial^{\alpha+\beta} / \partial t^{\alpha} \partial x^{\beta}$ (here $\alpha, \beta$ are nonnegative integers, $2 \alpha+\beta \leq 2$ ) on $Q_{t_{*}}$ and these derivatives can be continuously extended to $\bar{Q}_{t_{*}}$ [7].

DEFINITION 3.1. Classical solution of the boundary-value problem (2.9), (2.10), (2.11), and (2.12) is a function $c(t, x) \in C^{1,2}\left(\bar{Q}_{t_{*}}\right)$, which is symmetrical (2.12) and satisfies the diffusion equation (2.9) in $Q_{t_{*}}$ with initial data (2.10) and dynamical boundary condition (2.11). 
Let $A(t)=c_{0}(t)=c(t, 0)$ and assume that classical solution exists. We consider a function $c^{0}(t, x)=c(t, x)-A(t)$. Obviously, $c^{0}(t, 0)=c^{0}(t, \ell)=0$, $c_{x}^{0}(t, 0)=-c_{x}^{0}(t, \ell)$. It can be extended oddly to $[-\ell, \ell]$ and thus periodically on $\mathbb{R}^{1}$. Then, $c^{0}(t, \cdot) \in C^{1}\left(\mathbb{R}^{1}\right)$ and on the segment of interest $[0, \ell]$ it can be expanded to uniformly converging Fourier series by sine. Thus, it is possible to try to find the solution in $Q_{t_{*}}$ as

$$
c(t, x)=A(t)+\sum_{n=1}^{\infty} K_{n}(t) \sin \left(\frac{\pi n x}{\ell}\right) .
$$

Formally substitution $c(t, x)$ to the diffusion equation (2.9) gives

$$
\sum_{n=1}^{\infty}\left(\dot{K}_{n}(t)+\frac{K_{n}(t) \pi^{2} n^{2}}{\ell^{2}}\right) \sin \left(\frac{\pi n x}{\ell}\right)=-\dot{A}(t) .
$$

Making a scalar product in $L_{2}[0, \ell]$ of $(3.2)$ and $\sin (\pi n x / \ell)$, we obtain the system of differential equations for $K_{n}(t)$

$$
\begin{aligned}
& \dot{K}_{n}(t)+\frac{K_{n}(t) \pi^{2} n^{2}}{\ell^{2}}=-\frac{4 \dot{A}(t)}{\pi n}, \quad n=2 k-1, \\
& \dot{K}_{n}(t)+\frac{K_{n}(t) \pi^{2} n^{2}}{\ell^{2}}=0, \quad n=2 k, k=1,2,3, \ldots .
\end{aligned}
$$

Initial conditions come from fixing $t=0$ in (3.1), and from initial data (2.10) we see that $K_{n}(0)=0, K_{n}(t) \equiv 0$ if $n=2 k$, and for $n=2 k-1$

$$
K_{n}(t)=-\frac{4}{\pi n} \int_{0}^{t} \dot{A}(\tau) \varepsilon_{n}(t-\tau) d \tau, \quad \varepsilon_{n}(t)=\exp \left\{-\left(\frac{\pi n}{\ell}\right)^{2} t\right\} .
$$

Later in this paper, the symbol $\sum^{\prime}$ will be used as a sum by odd natural $n$. Obviously, $c(t, x)=c(t, \ell-x)$ and only boundary condition (2.11) is unsatisfied (formally yet). After substituting (3.1) into (2.11), assuming that series can be differentiated term by term, we obtain the main equation for $A(t)$

$$
\begin{gathered}
\dot{A}(t)=-\alpha_{1}(t) A^{2}(t)+\alpha_{2}(t) A(t)-\alpha_{3}(t) \sum^{\prime} \int_{0}^{t} \dot{A}(\tau) \varepsilon_{n}(t-\tau) d \tau, \\
\alpha_{1}(t)=\frac{b}{D g}, \quad \alpha_{2}(t)=\frac{\dot{g}}{g}, \quad \alpha_{3}(t)=\frac{4 g}{\ell}, \\
\varepsilon_{n}(t)=\exp \left\{-\left(\frac{\pi n}{\ell}\right)^{2} t\right\}, \quad \sum^{\prime}=\sum_{n=1,3,5, \ldots} .
\end{gathered}
$$

DEFINITION 3.2. The solution of (3.5) on segment $I=\left[0, t^{+}\right]$is a function $A(t) \in C^{1}(I)$, which satisfies (3.5) for all $t \in I$ as well as initial condition $A(0)=$ $\bar{c}_{0}$. The series in the right part converge for all $t \in I$, derivatives on the ends of $I$ are left or right. 
Specificity of this equation is in the term $\Sigma^{\prime} \int_{0}^{t}$. If, instead of it, there was a function of time only, not depending on $A$, it would be a Riccati equation, which is well studied in the theory of differential equations. The derivative $\dot{A}$ is present in both parts of the equation. It is impossible to use integration by parts (to remove $\dot{A}$ ) for one of the series will become divergent. Here appears an analogy with functional differential equations of neutral type [6]. Due to divergence, it is impossible to interchange an integral and a sum in the right part of (3.5). All this makes the study of (3.5) an interesting mathematical problem.

It is important to note that if there exists a solution on $I$, then series

$$
\sum^{\prime} \int_{0}^{t} \dot{A}(\tau) \varepsilon_{n}(t-\tau) d \tau
$$

converges on $I$ uniformly and absolutely as $|\dot{A}| \leq L$ (is limited),

$$
\left|\int_{0}^{t} \dot{A}(\tau) \varepsilon_{n}(t-\tau) d \tau\right| \leq L \int_{0}^{t} \varepsilon_{n}(t-\tau) d \tau \leq \frac{L \ell^{2}}{\pi^{2} n^{2}} .
$$

This numerical series converges. The value of the sum is estimated by $L \ell^{2} / 8$.

The initial boundary-value problem is reduced to this integrodifferential equation (3.5) in the following sense. Assume that the solution $A(t)$ exists on $I=\left[0, t^{+}\right]$. We define the following boundary-value problems:

$$
c_{t}(t, x)=c_{x x}(t, x), \quad c(0, x)=\bar{c}_{0}, \quad c(t, 0)=c(t, \ell)=A(t) .
$$

Such problems are well studied in [7]. The symmetry of initial and boundary conditions implies that $c(t, x)=c(t, \ell-x)$. Classical solution which exists is unique and can be found as a convergent in $C^{1,2}$ trigonometric series. Thus a formal series (3.1), built earlier, will present a classical solution and all operations at it were legal.

4. Obtaining solution $A(t)$. Equation (3.5) differs from Riccati equation by the fact that instead of differential operator $d / d t$ the integrodifferential one is present, containing a series $\Sigma^{\prime}$. So we consider a functional differential problem on $I=\left[0, t^{+}\right]$

$$
\begin{gathered}
\dot{A}(t)+\alpha_{3}(t) \sum^{\prime} \int_{0}^{t} \dot{A}(\tau) \varepsilon_{n}(t-\tau) d \tau=f(t), \\
A(0)=\bar{c}_{0}, \quad \alpha_{3}=\frac{4 g}{\ell} \in C^{1}(I), \quad f \in C(I) .
\end{gathered}
$$

Let $B(t)=\dot{A}(t)$ and define an iterative process

$$
B_{0}(t)=0 \quad\left(A_{0}(t)=\bar{c}_{0}\right), \quad B_{k+1}(t)+\alpha_{3}(t) \sum^{\prime} \int_{0}^{t} B_{k}(\tau) \varepsilon_{n}(t-\tau) d \tau=f(t) .
$$


In case $B_{k}$ is continuous, $\left|B_{k}\right| \leq L_{k}$ on $I$ and $\sum^{\prime}$ converges absolutely and uniformly (being majorized by a convergent series as explained above). Due to $B_{0}, B_{1}=f \in C(I)$, one can obtain that a sequence $B_{k}(t)$ on $I$ is defined correctly and $B_{k} \in C(I)$.

We now study the convergence. Consider a series $B_{0}+\left(B_{1}-B_{0}\right)+\left(B_{2}-B_{1}\right)+$ ... which is equal to the sequence $B_{k}$. Later, we will use a norm $C(I)$ : $\|\cdot\|=$ $\|\cdot\|_{C(I)}$. The following estimations are true:

$$
\begin{gathered}
\left\|B_{0}\right\|=0, \quad\left\|B_{1}-B_{0}\right\|=\|f\|, \\
\left|B_{2}(t)-B_{1}(t)\right|=\left|\alpha_{3}(t) \sum^{\prime} \int_{0}^{t} f(\tau) \varepsilon_{n}(t-\tau) d \tau\right| \leq\left\|\alpha_{3}\right\| \cdot\|f\| \cdot \Psi(t),
\end{gathered}
$$

where

$$
\Psi(t)=\sum^{\prime} \int_{0}^{t} \varepsilon_{n}(t-\tau) d \tau=\sum^{\prime} \frac{\ell^{2}}{(n \pi)^{2}}\left[1-\exp \left\{-\left(\frac{n \pi}{\ell}\right)^{2} t\right\}\right] .
$$

A series for $\Psi$ on $I$ is majorized by $\sum^{\prime} \ell^{2} /(\pi n)^{2}$. A function $\Psi(t)$ has the following properties: $\Psi(0)=0, \Psi(t)>0$ when $t>0, \Psi(t)$ grows on $t$, and $\Psi(t) \leq \ell^{2} / 8$. Each term is continuous, so $\Psi \in C(I)$ and $\|\Psi\|=\Psi\left(t^{+}\right)$.

We have obtained an estimation $\left\|B_{2}-B_{1}\right\| \leq\left\|\alpha_{3}\right\| \cdot\|f\| \cdot \Psi\left(t^{+}\right)$. Now, only local solution will be constructed since its continuation is a subject of a special study. Let $t^{+}$be such that $\left\|\alpha_{3}\right\| \Psi\left(t^{+}\right) \leq r<1$. Then,

$$
\begin{aligned}
\left\|B_{2}-B_{1}\right\| & \leq r\|f\|, \\
\left\|B_{3}-B_{2}\right\| & \leq\left|\alpha_{3}(t) \sum^{\prime} \int_{0}^{t}\right| B_{2}(\tau)-B_{1}(\tau)\left|\varepsilon_{n}(t-\tau) d \tau\right| \\
& \leq\left\|\alpha_{3}\right\| \cdot\left\|B_{2}-B_{1}\right\| \cdot \Psi\left(t^{+}\right) \leq r\left\|B_{2}-B_{1}\right\| \leq r^{2}\|f\| .
\end{aligned}
$$

Continuing this process, one obtains $B_{k} \rightrightarrows B \in C(I)$ and

$$
\|B\| \leq\left\|B_{0}\right\|+\left\|B_{1}-B_{0}\right\|+\cdots \leq \rho\|f\|, \quad \rho=\frac{1}{1-r} .
$$

Estimation (4.6) implies continuous dependence $B=\dot{A}$ of $f$.

THEOREM 4.1. For sufficiently small $t^{+}\left(\left\|\alpha_{3}\right\| \cdot \Psi\left(t^{+}\right)<1\right)$, the unique solution $A \in C^{1}(I)$ of (4.1) exists for all $f \in C(I)$.

The existence is proved, $A(t)=\bar{c}_{0}+\int_{0}^{t} B(\tau) d \tau$. Suppose that there exists one more solution $F \in C^{1}(I)$. Using linearity of (4.1), from (4.6), one obtains $\|B-\dot{F}\|=0$, which means that $A=F$ on $I$. If $F$ exists on a smaller segment $J=\left[0, t^{0}\right]$, then $t^{+}$is reduced to $t^{0}(\Psi(t) \rightarrow 0$ monotonically when $t \rightarrow 0)$. Then, $A=F$ on $J$ and a solution $A$ can be considered as continuation of $F$ from $J$ to $I$. 
REMARK 4.2. The condition $\left\|\alpha_{3}\right\| \cdot \Psi\left(t^{+}\right)<1$ is true without any limitations on $t^{+}$if $\|g\|<2 / \ell$, for $\alpha_{3}=4 \mathfrak{g} / \ell, \Psi \leq \ell^{2} / 8$. These limitations are tributes to the method of contractive mappings.

THEOREM 4.3. The unique solution $A \in C^{1}(I)$ of (4.1) with continuous right part $f$ exists on any segment $I=\left[0, t^{+}\right]$, the following estimation is true: $\|\dot{A}\| \leq$ $R\|f\|$.

Proof. As $T(t) \in\left[T^{-}, T^{+}\right]$, we choose $t_{1}^{+}$so that the following inequality holds: $\left\|\alpha_{3}\right\| \cdot \Psi\left(t_{1}^{+}\right) \leq r<1$. The solution will be constructed on $I_{1}=\left[0, t_{1}^{+}\right]$in accordance with Theorem 4.1. From (4.6), it follows that $\|\dot{A}\|_{I_{1}} \leq(1-r)^{-1}\|f\|_{I_{1}}$. We consider (4.1) on the next segment $I_{2}=\left[t_{1}^{+}, 2 t_{1}^{+}\right]$with initial data $A\left(t_{1}^{+}\right)$

$$
\begin{gathered}
\dot{A}(t)+\alpha_{3}(t) \sum^{\prime} \int_{t_{1}+}^{t} \dot{A}(\tau) \varepsilon_{n}(-\tau) d \tau=\tilde{f}(t), \\
\tilde{f}(t)=f(t)-\alpha_{3}(t) \sum^{\prime} \varepsilon_{n}(t) \int_{0}^{t_{1}+} \dot{A}(\tau) \varepsilon_{n}(-\tau) d \tau .
\end{gathered}
$$

Noting that

$$
\varepsilon_{n}(t)=\varepsilon_{n}\left(t-t_{1}^{+}\right) \varepsilon_{n}\left(t_{1}^{+}\right), \quad \varepsilon_{n}(-\tau)=\varepsilon_{n}\left(t_{1}^{+}-\tau\right) \varepsilon_{n}\left(-t_{1}^{+}\right), \quad \varepsilon_{n}(0)=1,
$$

and moving the origin to $t_{1}^{+}$, one obtains problem (4.1) with modified right part. Estimation (4.6) implies that $\|\dot{A}\|_{I_{2}} \leq \rho\|\tilde{f}\|_{I_{2}}$. We estimate $\|\tilde{f}\|_{I_{2}}$ as follows:

$$
\begin{aligned}
|\tilde{f}(t)| & \leq|f(t)|+\left|\alpha_{3}(t)\right| \sum^{\prime} \varepsilon_{n}\left(t-t_{1}^{+}\right) \varepsilon_{n}\left(t_{1}^{+}\right) \int_{0}^{t_{1}^{+}}|\dot{A}(\tau)| \varepsilon_{n}(-\tau) d \tau \\
& \leq|f(t)|+\left\|\alpha_{3}\right\|_{I_{2}} \cdot\|\dot{A}\|_{I_{1}} \cdot \Psi\left(t_{1}^{+}\right), \quad 0<\varepsilon_{n}\left(t-t_{1}^{+}\right) \leq 1, t \in I_{2}, \\
\|\tilde{f}\|_{I_{2}} & \leq\|f\|_{I_{2}}+r\|\dot{A}\|_{I_{1}} \leq\|f\|_{I_{2}}+r(1-r)^{-1}\|f\|_{I_{1}} .
\end{aligned}
$$

From here, the following is easily obtained:

$$
\begin{aligned}
\|\dot{A}\|_{I_{2}} & \leq \rho\|\tilde{f}\|_{I_{2}} \leq \rho\left(\|f\|_{I_{2}}+r \rho\|f\|_{I_{1}}\right) \\
& \leq \rho(1+r \rho)\|f\|_{I_{1} \cup I_{2}}=\rho^{2}\|f\|_{I_{1} \cup I_{2}} .
\end{aligned}
$$

Comparing this result with $\|\dot{A}\|_{I_{1}} \leq(1-r)^{-1}\|f\|_{I_{1}}, 0<r<1$, we have the following:

$$
\|\dot{A}\|_{I_{1} \cup I_{2}} \leq \rho^{2}\|f\|_{I_{1} \cup I_{2}}, \quad \rho=\frac{1}{(1-r)} .
$$

In the same way, one can consider the next segment $I_{3}=\left[2 t_{1}^{+}, 3 t_{1}^{+}\right]$

$$
\begin{aligned}
& \dot{A}(t)+\alpha_{3}(t) \sum^{\prime} \varepsilon_{n}(t) \int_{2 t_{1}^{+}}^{t} \dot{A}(\tau) \varepsilon_{n}(-\tau) d \tau=\hat{f}(t), \\
& \hat{f}(t)=f(t)-\alpha_{3}(t) \sum^{\prime} \varepsilon_{n}(t) \int_{0}^{2 t_{1}^{+}} \dot{A}(\tau) \varepsilon_{n}(-\tau) d \tau .
\end{aligned}
$$


Using the same technique, the following estimation is obtained:

$$
\|\dot{A}\|_{I_{1} \cup I_{2} \cup I_{3}} \leq(1-r)^{-3}\|f\|_{I_{1} \cup I_{2} \cup I_{3}} .
$$

In this way, the continuous function $A(t)$ on $I$ is constructed. On any segment with length $t_{1}^{+}$, it satisfies (4.1). The way of construction guarantees that discontinuities of $\dot{A}(t)$ can be only of the one kind and can exist only on the ends of the segments. But even in this case all terms in $\sum^{\prime}$ are continuous, and boundness of $|\dot{A}|$ on $I$ implies absolute and uniform convergence. Thus continuity of the second term of (4.1) implies the continuity of the derivative $\dot{A}$, which means that $A \in C^{1}(I)$. The uniqueness of the solution follows from the way of constructing consequently on $I_{1}, I_{2}, \ldots$. The estimation is true on the segment $I$ : $\|\dot{A}\| \leq R\|f\|, R=(1-r)^{-N}$.

Now, remember that in the initial equation (3.5), square function $\alpha_{1}(t) A^{2}(t)$ $+\alpha_{2}(t) A(t)$ is instead of $f(t)$. We consider a new iterative process $A_{0}(t)=\bar{c}_{0}$, $B_{0}(t)=0$,

$$
\begin{array}{r}
B_{k+1}(t)+\alpha_{3}(t) \sum^{\prime} \int_{0}^{t} B_{k+1}(\tau) \varepsilon_{n}(t-\tau) d \tau \\
=-\alpha_{1}(t) A_{k}^{2}(t)+\alpha_{2}(t) A_{k}(t)
\end{array}
$$

$A_{k+1}(t)=\bar{c}_{0}+\int_{0}^{t} B_{k+1}(\tau) d \tau$, which is the same with

$$
\begin{array}{r}
\dot{A}_{k+1}(t)+\alpha_{3}(t) \sum^{\prime} \int_{0}^{t} \dot{A}_{k+1}(\tau) \varepsilon_{n}(t-\tau) d \tau \\
=-\alpha_{1}(t) A_{k}^{2}(t)+\alpha_{2}(t) A_{k}(t) .
\end{array}
$$

The sequences $B_{k} \in C(I)$ and $A_{k} \in C^{1}(I)$ are defined correctly on any given segment $\left[0, t^{+}\right]$. The solutions $B_{k+1}$ with given $A_{k}$ are defined by (4.1) (which is linear with respect to unknown function $B_{k+1}$ ) -it follows from Theorems 4.1 and 4.3.

THEOREM 4.4. When $t^{+}$is small enough, $B_{k}$ is bounded, that is, the following estimation holds: $\left\|B_{k}\right\|_{C(I)} \leq M=$ const.

Proof. Let time instant $t^{+}$be chosen such that both inequalities $\left\|\alpha_{3}\right\|_{I}$. $\Psi\left(t^{+}\right) \leq r<1$ and (4.6) are true. By the way, on the initial stage there is no need to bound $t^{+}$due to Theorem $4.3(\|\dot{A}\| \leq R\|f\|)$. With respect to (4.14), one obtains $\left(\alpha_{4}=-\alpha_{1} \bar{c}_{0}^{2}+\alpha_{2} \bar{c}_{0}, \alpha_{5}=\alpha_{2}-2 \alpha_{1} \bar{c}_{0}\right)$

$$
\begin{aligned}
\left\|B_{k+1}\right\|_{I} & \leq \rho\left\|-\alpha_{1} A_{k}^{2}+\alpha_{2} A_{k}\right\|_{I} \\
& =\rho\left\|\alpha_{4}+\alpha_{5} \int_{0}^{t} B_{k} d \tau-\alpha_{1}\left(\int_{0}^{t} B_{k} d \tau\right)^{2}\right\|_{I} \\
& \leq \rho\left[\left\|\alpha_{4}\right\|_{I}+t^{+}\left\|\alpha_{5}\right\|_{I} \cdot\left\|B_{k}\right\|_{I}+t^{+2}\left\|\alpha_{1}\right\|_{I} \cdot\left\|B_{k}\right\|_{I}^{2}\right] .
\end{aligned}
$$


Thus, the following estimation is obtained:

$$
\left\|B_{k+1}\right\| \leq \beta_{0}+\beta_{1} t^{+}\left\|B_{k}\right\|+\beta_{2} t^{+2}\left\|B_{k}\right\|^{2} .
$$

Note that while $t^{+}$becomes smaller, constants $\beta_{i}$ cannot grow, yet stay positive.

Consider the square function $f(x)=\beta_{1} t^{+} x+\beta_{2} t^{+2} x^{2}, x \geq 0$. For any condition $0 \leq x \leq M$, it is possible to take $t^{+} \leq \varepsilon$ so small so that $0 \leq f(x) \leq \gamma x$, $0<\gamma<1$. For instance, $\beta_{1} t^{+} \leq \gamma / 2$ and $\beta_{2} t^{+2} M \leq \gamma / 2$.

Let $t^{+}$be so small so that the following inequalities are true:

$$
\left\|\alpha_{3}\right\| \cdot \Psi\left(t^{+}\right) \leq r<1, \quad\left\|B_{k+1}\right\| \leq \beta_{0}+\gamma\left\|B_{k}\right\|, \quad 0<\gamma<1,\left\|B_{k}\right\| \leq M .
$$

It is important here to note that the inequality for $B_{k+1}$ is written assuming that $\left\|B_{k}\right\| \leq M$. Constant $M$, which can be made bigger reducing $t^{+}$, will be specified later.

As $B_{0}=0,\left\|B_{1}\right\| \leq \beta_{0}$. Quantity $\beta_{0}=\rho\left\|\alpha_{4}\right\|$ cannot grow while $t^{+}$reduces, but at the same time does not tend to be zero. Let $M>\beta_{0}$ (this can be obtained using $t^{+}$). Then,

$$
\left\|B_{2}\right\| \leq \beta_{0}+\gamma\left\|B_{1}\right\| \leq \beta_{0}+\gamma \beta_{0} .
$$

If $\beta_{0}+\gamma \beta_{0}<M$, then it would be possible to continue a simpler estimation

$$
\left\|B_{3}\right\| \leq \beta_{0}+\gamma\left\|B_{2}\right\| \leq \beta_{0}+\gamma \beta_{0}+\gamma^{2} \beta_{0} .
$$

Note that if choosing small enough $t^{+}$, the following is made true (for instance, if $M=1 / t^{+}$):

$$
\beta_{0}\left(1+\gamma+\gamma^{2}+\cdots\right)=\beta_{0}(1-\gamma)^{-1} \leq M
$$

then all simplified estimations will be true and $\left\|B_{k}\right\| \leq M$ for all $k \geq 0$.

REMARK 4.5. The choice $t^{+}$is constructive. We consider the simplest case. Choose $r<1$ and $t^{+}$from condition $4 g\left(t^{+}\right) \Psi\left(t^{+}\right) / \ell \leq r$. Series for $\Psi(t)$ converges quickly. Calculate $\beta_{i}$ with given $r, t^{+}$, known initial concentration $\bar{c}_{0}$, and coefficients $D, g$, and $b$. Then, for some $\gamma \in(0,1)$, by reducing $t^{+}$, if necessary, we obtain

$$
\beta_{1} t^{+} \leq \frac{\gamma}{2}, \quad \beta_{2} t^{+} \leq \frac{\gamma}{2}, \quad M=\frac{1}{t^{+}} \geq \beta_{0}(1-\gamma)^{-1}
$$

After that, it is possible to come back to old times.

THEOREM 4.6. For a small enough $t^{+}$, the unique solution $A \in C^{1}(I)$ of the initial functional differential equation (3.5) on a segment $I=\left[0, t^{+}\right]$exists. 
Proof. The following is obtained from (4.14) using $A_{k}(0)=\bar{c}_{0}, \dot{A}_{k}=B_{k}$ :

$$
\begin{gathered}
B_{k+2}(t)-B_{k+1}(t)+\alpha_{3}(t) \sum^{\prime} \int_{0}^{t}\left(B_{k+2}(\tau)-B_{k+1}(\tau)\right) \varepsilon_{n}(t-\tau) d \tau \\
-\alpha_{1}(t)\left(A_{k+1}^{2}(t)-A_{k}^{2}(t)\right)+\alpha_{2}(t)\left(A_{k+1}(t)-A_{k}(t)\right) \\
=\left\{\alpha_{5}-\alpha_{1} \int_{0}^{t}\left(B_{k+1}-B_{k}\right) d \tau\right\} \cdot \int_{0}^{t}\left(B_{k+1}-B_{k}\right) d \tau .
\end{gathered}
$$

Let $t^{+}$be so small so that from Theorem 4.4

$$
\left\|\alpha_{3}\right\|_{I} \cdot \Psi\left(t^{+}\right) \leq r<1, \quad\left\|B_{k}\right\|_{I} \leq M=\frac{1}{t^{+}} .
$$

Using the estimation (4.6) from (4.1),

$$
\left\|B_{k+2}-B_{k+1}\right\| \leq \rho\left\{\left\|\alpha_{5}\right\|+2\left\|\alpha_{1}\right\| M t^{+}\right\} \cdot\left\|B_{k+1}-B_{k}\right\| t^{+}=\beta t^{+}\left\|B_{k+1}-B_{k}\right\|
$$

is obtained. Choose $\beta$ and obtain the contraction (reducing $t^{+}$)

$$
\left\|B_{k+2}-B_{k+1}\right\| \leq s\left\|B_{k+1}-B_{k}\right\|, \quad 0<s<1 .
$$

Then, the well-known method of contracting mappings is used to prove the existence of unique solution $A \in C^{1}(I)$ to (3.5). Its derivative $B(t)=\dot{A}(t)$ can be estimated $\left(B_{0}=0\right):\|B\| \leq\left\|B_{1}\right\| /(1-s)$.

5. Numerical results. Difference schemes with fourth-order approximation $\left(O\left(h^{4}\right)\right.$, where $h$ is the spatial step) are constructed for numerical experiments with the model. The stability is studied in [2]. The desorption flux curves have been calculated for different initial data and parameters. The curves are quite close to those obtained from physical experiments.

Local maximum points of the curve $J(t)$ (density of desorption flux) are of interest. On Figure 5.1 there are three plots for the values (one after another) in Table 5.1.

For all plots, the flux is items per $\mathrm{cm}^{2}$ per second.

The first maximum appears because the rates of diffusion and desorption grow together with temperature. Then the decrease of the gas amount in the plate implies lowering of the curve. Existence of the second maximum (note that gas interaction with traps is not taken into consideration) is explained by difference between rates of the processes on surface and in depth. Quick decrease of the surface concentration $q(t)$ implies big gradient of volume concentration $c(t, x)$ near $x=0$, which defines a significant diffusion flux towards the surface. Desorption flux quickly decreases, but later, because of arraying gas, it increases again, forming the second maximum. 


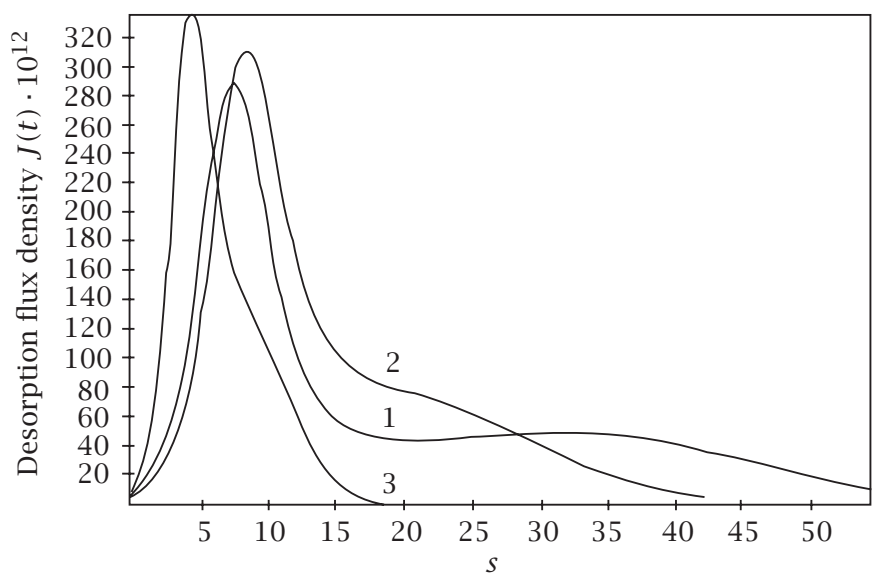

FIGURE 5.1

TABLE 5.1

\begin{tabular}{lllr}
\hline$D_{0}=5 \cdot 10^{-3} \mathrm{~cm}^{2} / \mathrm{s}$ & $g_{0}=100 \mathrm{~cm}^{-1}$ & $b_{0}=0.12 \mathrm{~cm}^{2} / \mathrm{s}$ & $\dot{T}=10 \mathrm{~K} / \mathrm{s}$ \\
$E_{D}=20 \mathrm{~kJ} / \mathrm{mol}$ & $E_{g}=10^{-3} \mathrm{~kJ} / \mathrm{mol}$ & $E_{b}=84 \mathrm{~kJ} / \mathrm{mol}$ & $T_{0}=279 \mathrm{~K}$ \\
\hline$D_{0}=5 \cdot 10^{-3} \mathrm{~cm}^{2} / \mathrm{s}$ & $g_{0}=100 \mathrm{~cm}^{-1}$ & $b_{0}=0.12 \mathrm{~cm}^{2} / \mathrm{s}$ & $\dot{T}=10 \mathrm{~K} / \mathrm{s}$ \\
$E_{D}=16 \mathrm{~kJ} / \mathrm{mol}$ & $E_{g}=10^{-3} \mathrm{~kJ} / \mathrm{mol}$ & $E_{b}=85 \mathrm{~kJ} / \mathrm{mol}^{2}$ & $T_{0}=279 \mathrm{~K}$ \\
\hline$D_{0}=5 \cdot 10^{-2} \mathrm{~cm}^{2} / \mathrm{s}$ & $g_{0}=100 \mathrm{~cm}^{-1}$ & $b_{0}=0.12 \mathrm{~cm}^{2} / \mathrm{s}$ & $\dot{T}=10 \mathrm{~K} / \mathrm{s}$ \\
$E_{D}=20 \mathrm{~kJ} / \mathrm{mol}$ & $E_{g}=10^{-3} \mathrm{~kJ} / \mathrm{mol}$ & $E_{b}=84 \mathrm{~kJ} / \mathrm{mol}$ & $T_{0}=279 \mathrm{~K}$ \\
\hline
\end{tabular}

Here are some more examples of how the coefficients affect the curve $J(t)=$ $b(t) q^{2}(t)$. The curves on Figures $5.2,5.3,5.4,5.5$, and 5.6 differ by only one parameter, its values are given up-to-down, left-to-right with respect to the maximum $E_{D}=16,19,22 ; b_{0}=0.3,0.12,0.06 ; E_{b}=76,78,90 ; D_{0}=14 \mathrm{e}-3,5 \mathrm{e}-3,1 \mathrm{e}-3$; and $E_{g}=2,1,1$ e-3. Other parameters are given in Table 5.2.

TABLE 5.2

\begin{tabular}{lllr}
\hline$D_{0}=5 \cdot 10^{-3} \mathrm{~cm}^{2} / \mathrm{s}$ & $g_{0}=100 \mathrm{~cm}^{-1}$ & $b_{0}=0.12 \mathrm{~cm}^{2} / \mathrm{s}$ & $\dot{T}=10 \mathrm{~K} / \mathrm{s}$ \\
$E_{D}=20 \mathrm{~kJ} / \mathrm{mol}$ & $E_{g}=10^{-3} \mathrm{~kJ} / \mathrm{mol}$ & $E_{b}=84 \mathrm{~kJ} / \mathrm{mol}$ & $T_{0}=279 \mathrm{~K}$ \\
\hline
\end{tabular}

The influence of energy of activation of diffusion (it defines the exponential part of the Arrhenius law) is well seen. The difference is insignificant when temperatures are low (in the beginning of the experiment), but important for how gas leaves the plate; when the parameter is low, gas leaves quicker, but when high, then slower and fluently, and the second maximum appears. The coefficient $D(T(t))$ is the most difficult to vary as it appears in the stability conditions for the difference schemes. 


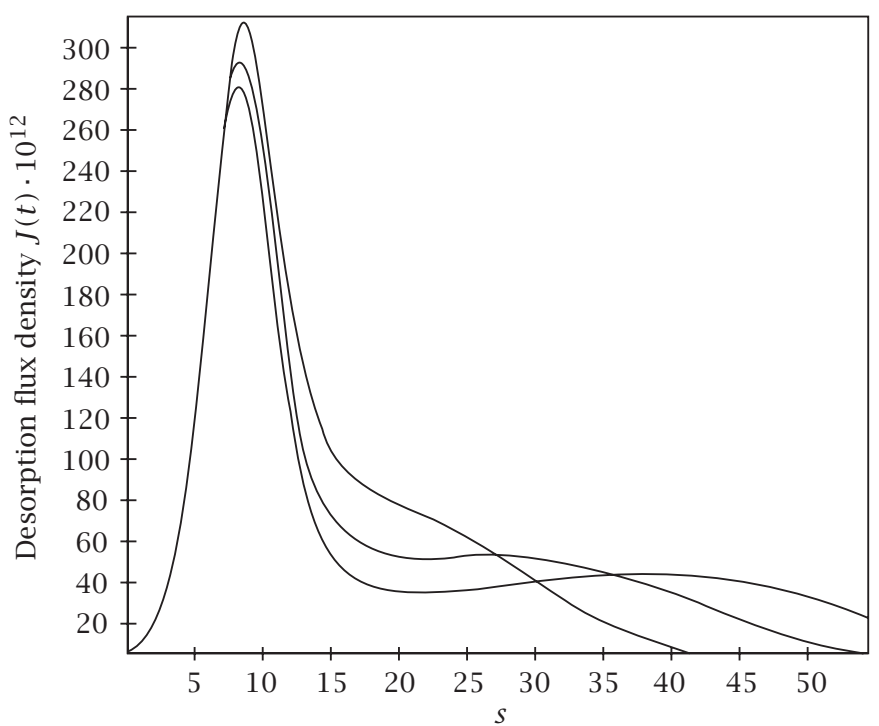

FIGURE 5.2

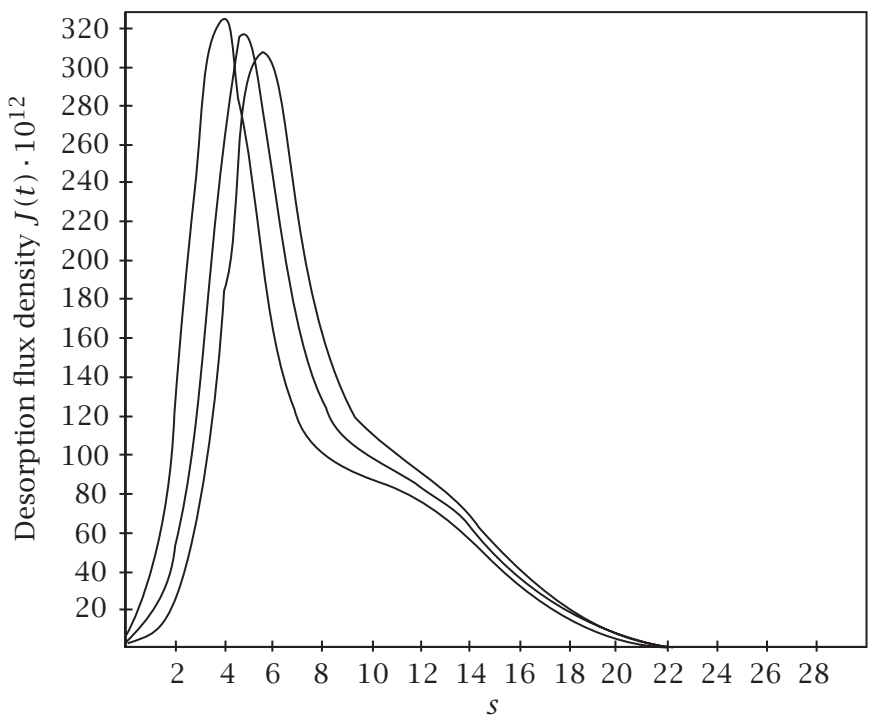

FIGURE 5.3

Note that the desorption coefficient $b$ nearly does not affect the end of the experiment. All three curves meet at the same point. Probably, at high temperatures, the exponential part of $b=b(T)$ "eats" any difference. The time of degassing is nearly the same $(\approx 22$ s on the upper plot and $\approx 20$ s on the lower). 


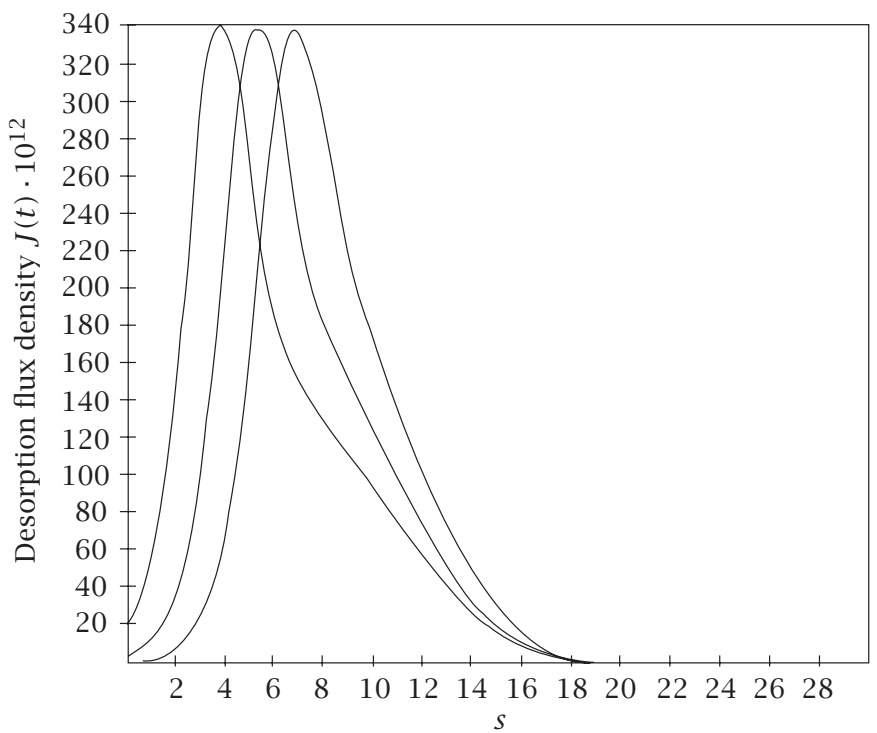

FIGURE 5.4

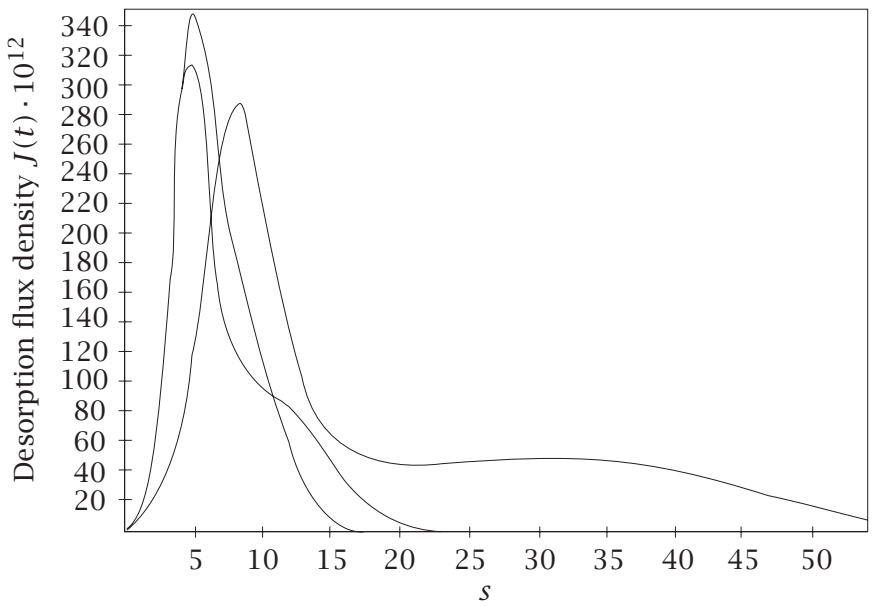

FIGURE 5.5

Now, we return to the problem of the second maximum of the flux. Illustrations given show that the most important for the second maximum appearance process is diffusion (thus parameters $D_{0}$ and $E_{D}$ ).

Consider the area below $J(t)$. Quantity $2 S I$, where $I=\int_{0}^{t^{*}} J(\tau) d \tau, t^{*} \gg 1$, is the number of hydrogen atoms, passing through both surfaces of the plate 


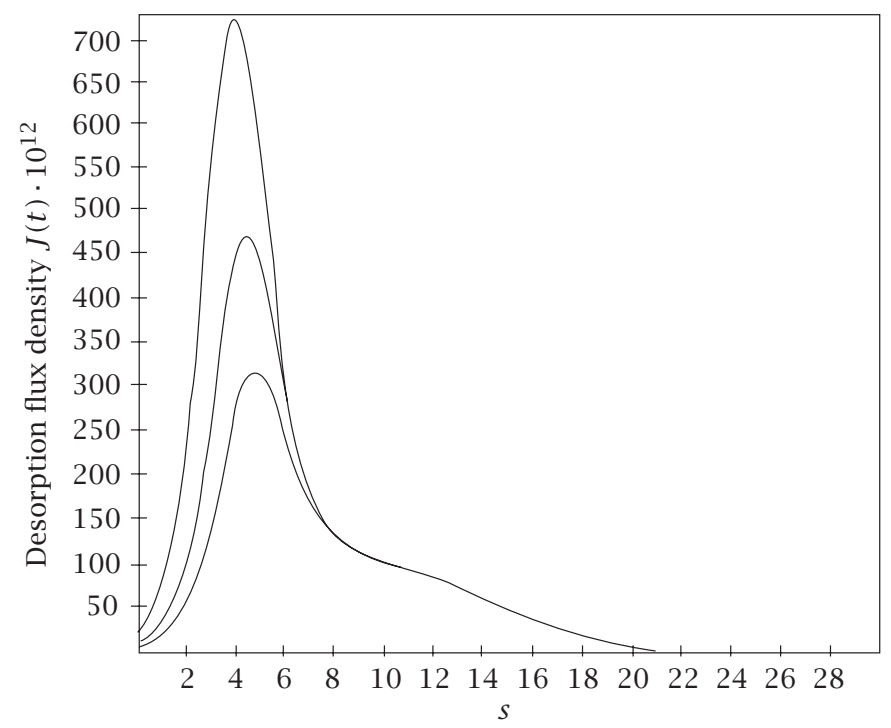

FIGURE 5.6

(each has the area $S$ ) during all the experiment. Initial amount of gas is defined by that dissolved in the volume $\left(c(0, x)=\bar{c}_{0}\right)$ and atoms on the surfaces $\left(q(0)=\bar{c}_{0} / g(0)\right)$. So, the following is true: $I=\bar{c}_{0} /(2 \ell)+\bar{c}_{0} / g(0)$. Then, it is obvious that any two curves $J(t)$ with equal parameters $g_{0}$ and $E_{g}$ must have the same area below the curve. With different $g_{0}$ and $E_{g}$ (and other parameters are equal), the areas will be noticeably different due to different amount of gas initially kept on the surface. And what is more, even if $g_{0}$ and $E_{g}$ are equal, parameter $g(0)$ in different experiments on different temperatures $T(0)$ will be different.

Parameter $g$ also influences the maximum value of the flux. As $J(t)=$ $b(t) q^{2}(t), q(t)=c(t, 0) / g(t)$, so the lower $g$-the greater number of atomswill go out to the surface at the unit time and later desorb. Note that considered values of $g$ nearly do not influence the last part of the experiment and its finish time $t^{*}$. But the maximum of $J$ "neatly" responds to $E_{g}$. This gives an opportunity to select the parameter $E_{g}$ only with the maximum value. Here are some illustrations.

Among the experimental curves, there are some curves with two humps, even the first is smaller than the second. It means that the second raise of the flux, conditioned by the delay of the "deep" amount of gas coming to the surface, is less significant than the first one, conditioned by growing rates of the processes. Such curves can be also obtained in the numerical experiment at special parameters values. On Figure 5.7 (parameters are in Table 5.3), there is an example: curves differ in the energies of activation of diffusion and desorption. 


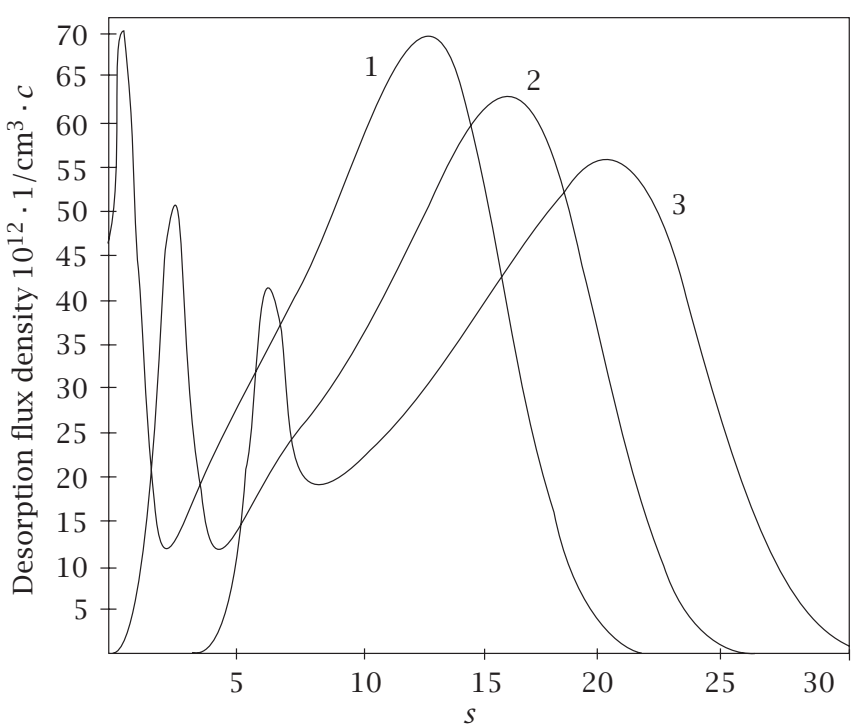

FIGURE 5.7

TABLE 5.3

\begin{tabular}{lllc}
\hline$D_{0}=5 \cdot 10^{-3} \mathrm{~cm}^{2} / \mathrm{s}$ & $g_{0}=800 \mathrm{~cm}^{-1}$ & $b_{0}=0.12 \mathrm{~cm}^{2} / \mathrm{s}$ & $\dot{T}=15 \mathrm{~K} / \mathrm{s}$ \\
$E_{D}=26,30,35 \mathrm{~kJ} / \mathrm{mol}$ & $E_{\mathfrak{g}}=10^{-6} \mathrm{~kJ} / \mathrm{mol}$ & $E_{b}=60,70,90 \mathrm{~kJ} / \mathrm{mol}$ & $T_{0}=270 \mathrm{~K}$ \\
\hline
\end{tabular}

The curves with three humps (also present in the experimental results) are hardly possible to be explained using only diffusion and surface processes. Although, such curves were also obtained in the considered model, taking the traps into consideration. The traps are different defects of the structure of the material, which can capture hydrogen and later release it, while the temperature grows. To consider traps, the model must be slightly modified

$$
\begin{aligned}
c_{t}(t, x) & =D(T) c_{x x}(t, x)-a_{1}(T) c(t, x)+a_{2}(T) z(t, x), \quad(t, x) \in Q_{t_{*}}, \\
z_{t}(t, x) & =a_{1}(T) c(t, x)-a_{2}(T) z(t, x), \quad T=T(t), \\
c(0, x) & =\bar{c}_{0}, \quad c(t, x)=c(t, \ell-x), \quad x \in[0, \ell], \\
c(t, 0) & =g(T) q(t), \quad \dot{q}(t)=-b(T) q^{2}(t)+D(T) c_{x}(t, 0) .
\end{aligned}
$$

Here, $a_{i}$ are the rates of capture $(i=1)$ and release $(i=2)$ of hydrogen by the traps. Their dependence on temperature is described by Arrhenius rule together with other parameters $a_{i}(t)=a_{i 0} \exp \left\{-E_{a_{i}} /[R T(t)]\right\}$. A function $z(t, x)$ is the concentration of hydrogen in the traps at the time $t$ in the point $x$. Below is an example of how the delay conditioned by the traps makes three 


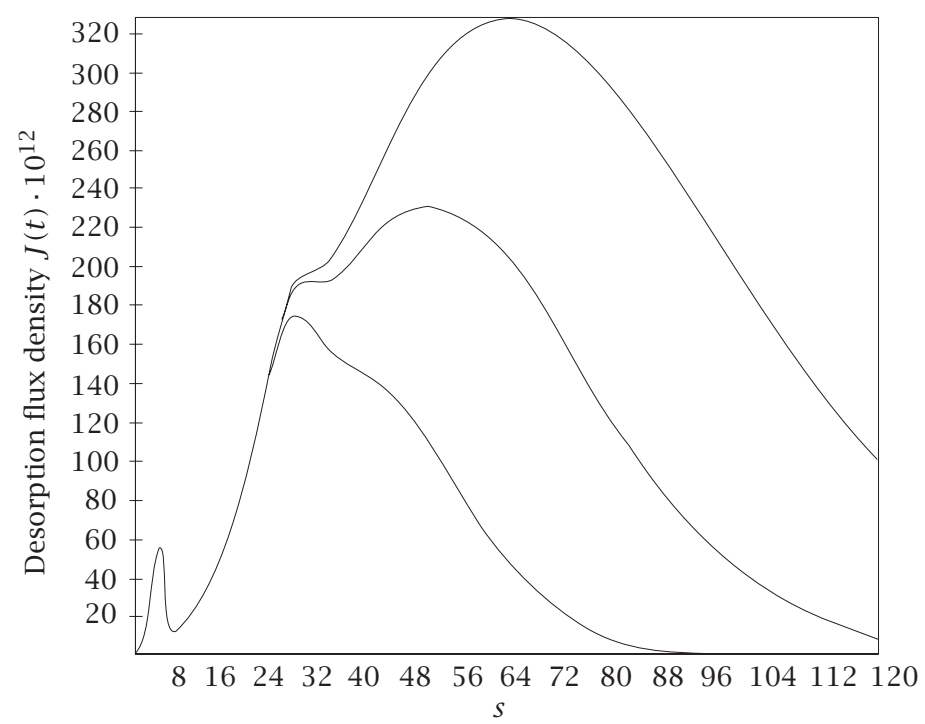

FIGURE 5.8

humps at the desorption flux curve. The common parameters for the three curves on Figure 5.8 are in Table $5.4, a_{2}=0.1,0.2,0.5$.

TABLE 5.4

\begin{tabular}{|c|c|c|c|}
\hline$D_{0}=5 \cdot 10^{-3} \mathrm{~cm}^{2} / \mathrm{s}$ & $g_{0}=800 \mathrm{~cm}^{-1}$ & $b_{0}=0.12 \mathrm{~cm}^{2} / \mathrm{s}$ & $\dot{T}=15 \mathrm{~K} / \mathrm{s}$ \\
\hline$E_{D}=30 \mathrm{~kJ} / \mathrm{mol}$ & $E_{\mathfrak{g}}=5 \cdot 10^{-2} \mathrm{~kJ} / \mathrm{mol}$ & $E_{b}=70 \mathrm{~kJ} / \mathrm{mol}$ & $T_{0}=230 \mathrm{~K}$ \\
\hline$a_{1}=10^{-3} \mathrm{~s}^{-1}$ & $E_{a_{1}}=0 \mathrm{~s}^{-1}$ & $E_{a_{2}}=15 \mathrm{~s}^{-1}$ & $t=120 \mathrm{~s}$ \\
\hline
\end{tabular}

Note that the time interval is taken significantly larger than that in the experiments without traps as, because of the delay conditioned by the traps, it takes more time for hydrogen to desorb. One more point to note is that at low temperatures the difference in the trapping rates is insignificant, yet at high temperatures even a small difference completely changes a curve.

Thus, numerical experiments corroborate the adequacy of the model.

\section{REFERENCES}

[1] G. Alefeld and J. Völkl (eds.), Hydrogen in Metals, Springer-Verlag, Berlin, 1978.

[2] I. A. Chernov, Mathematical modeling of gas transfer in solids, Trans. Inst. Appl. Math. Research of Karelian Research Centre RAS (1999), no. 1, 205-216.

[3] I. E. Gabis, The method of concentration pulses for studying hydrogen transport in solids, Technical Physics 44 (1999), no. 1, 90-94.

[4] I. E. Gabis, T. N. Kompaniets, and A. A. Kurdyumov, Surface processes and hydrogen permeability through metals, Interactions of Hydrogen with Metals (A. P. Zakharov, ed.), Nauka, Moscow, 1987, pp. 177-206. 
[5] I. E. Gabis, A. A. Kurdyumov, and N. A. Tikhonov, Equipment for complex investigation of gas interaction with metals, Vestn. St. Petersburg Univ., Ser. 4 (physics and chemistry) 2 (1993), no. 11, 77-79 (Russian).

[6] J. Hale, Theory of Functional Differential Equations, Applied Mathematical Sciences, vol. 3, Springer-Verlag, New York, 1977.

[7] V. P. Mikhailov, Partial Differential Equations, Nauka, Moscow, 1983 (Russian).

[8] Yu. V. Zaika, The solvability of the equations for a model of gas transfer through membranes with dynamic boundary conditions, Comput. Math. Math. Phys. 36 (1996), no. 12, 1731-1741.

[9]___ Determination of model parameters for the hydrogen permeability of metals, Technical Physics 43 (1998), no. 11, 1304-1308.

[10] _ Parametric identification of a model for hydrogen transfer through double-layer membranes, Technical Physics 45 (2000), no. 5, 554-562.

Yu. V. Zaika: Institute of Applied Mathematical Research, Karelian Research Centre, Petrozavodsk, Russia

E-mail address: zaika@krc.kare1ia.ru

I. A. Chernov: Institute of Applied Mathematical Research, Karelian Research Centre, Petrozavodsk, Russia

E-mail address: chernov@kare1ia.ru 


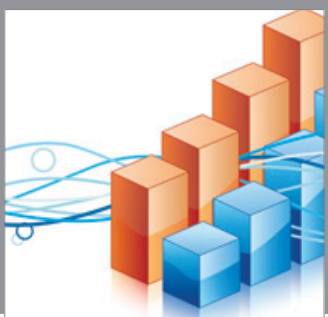

Advances in

Operations Research

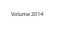

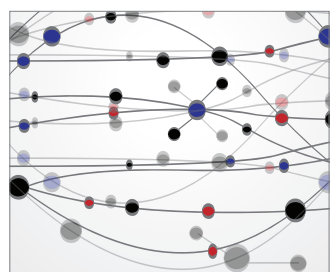

\section{The Scientific} World Journal
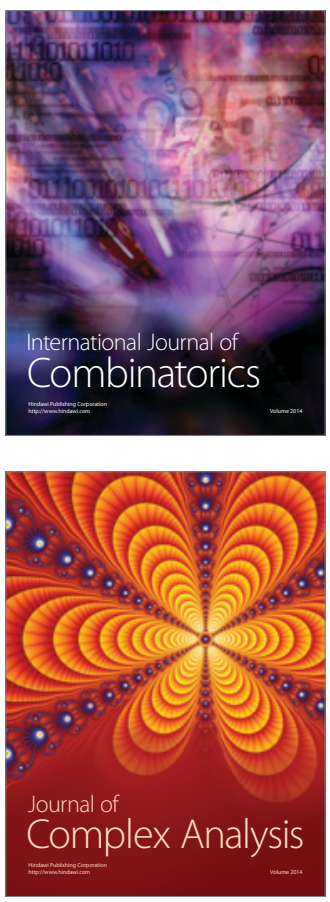

International Journal of

Mathematics and

Mathematical

Sciences
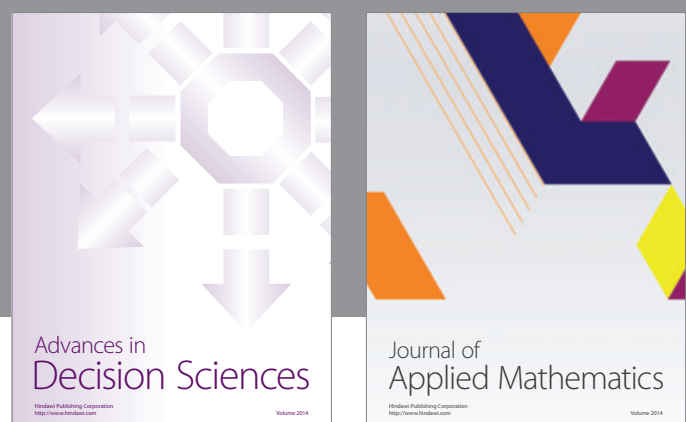

Journal of

Applied Mathematics
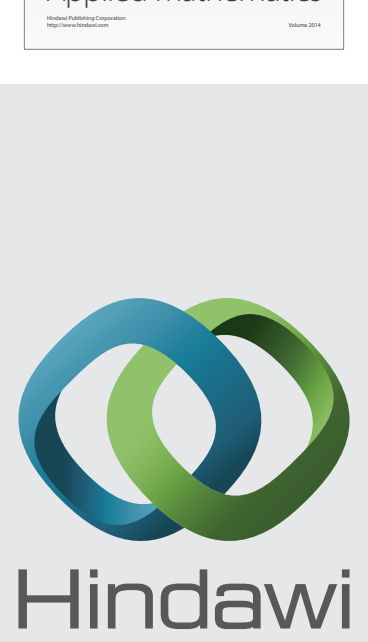

Submit your manuscripts at http://www.hindawi.com
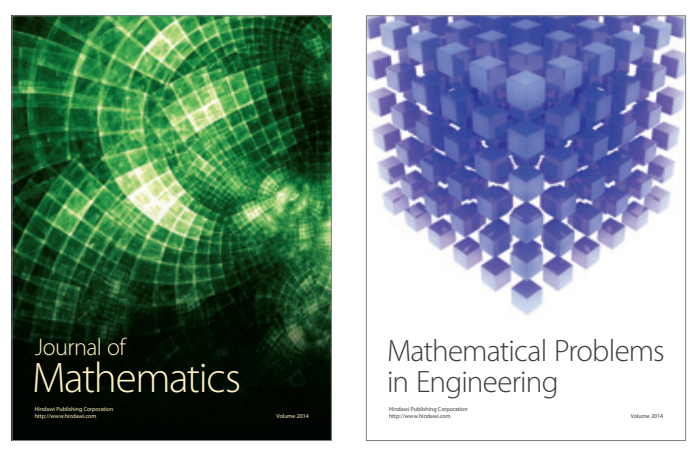

Mathematical Problems in Engineering
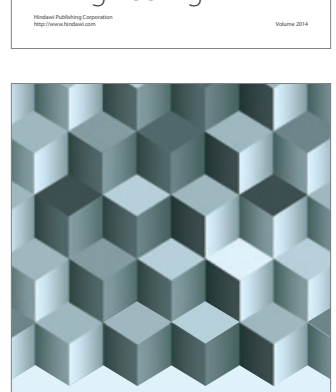

Journal of

Function Spaces
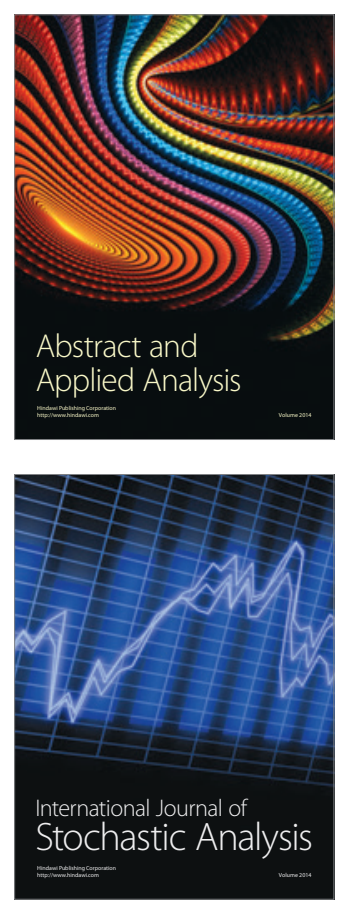

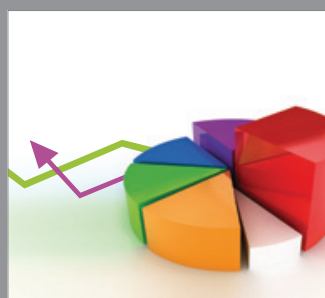

ournal of

Probability and Statistics

Promensencen
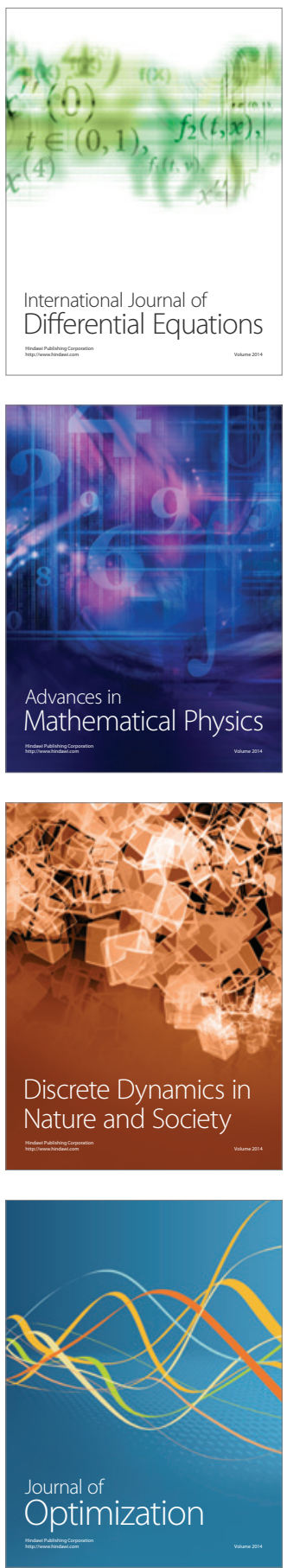